\title{
Statistical Power in Plant Pathology Research
}

\author{
David H. Gent, ${ }^{\dagger}$ Paul D. Esker, and Alissa B. Kriss
}

First author: U.S. Department of Agriculture-Agricultural Research Service, Forage Seed and Cereal Research Unit, and Oregon State University, Department of Botany and Plant Pathology, Corvallis 97331; second author: Department of Plant Pathology and Environmental Microbiology, Penn State University, University Park 16802; and third author: Syngenta Crop Protection, LLC, Greensboro, NC 27409. Accepted for publication 24 August 2017.

\begin{abstract}
In null hypothesis testing, failure to reject a null hypothesis may have two potential interpretations. One interpretation is that the treatments being evaluated do not have a significant effect, and a correct conclusion was reached in the analysis. Alternatively, a treatment effect may have existed but the conclusion of the study was that there was none. This is termed a Type II error, which is most likely to occur when studies lack sufficient statistical power to detect a treatment effect. In basic terms, the power of a study is the ability to identify a true effect through a statistical test. The power of a statistical test is 1 - (the probability of Type II errors), and depends on the size of treatment effect (termed the effect size), variance, sample size, and significance criterion (the probability of a Type I error, $\alpha$ ). Low statistical power is prevalent in scientific literature in general, including plant pathology. However, power is rarely reported, creating uncertainty in the interpretation of nonsignificant results and potentially underestimating small, yet biologically significant relationships. The appropriate level of power

for a study depends on the impact of Type I versus Type II errors and no single level of power is acceptable for all purposes. Nonetheless, by convention 0.8 is often considered an acceptable threshold and studies with power less than 0.5 generally should not be conducted if the results are to be conclusive. The emphasis on power analysis should be in the planning stages of an experiment. Commonly employed strategies to increase power include increasing sample sizes, selecting a less stringent threshold probability for Type I errors, increasing the hypothesized or detectable effect size, including as few treatment groups as possible, reducing measurement variability, and including relevant covariates in analyses. Power analysis will lead to more efficient use of resources and more precisely structured hypotheses, and may even indicate some studies should not be undertaken. However, the conclusions of adequately powered studies are less prone to erroneous conclusions and inflated estimates of treatment effectiveness, especially when effect sizes are small.
\end{abstract}

The use, limitations, and interpretation of $P$ values and null hypothesis testing in modern statistical inference continues to be discussed across statistics and other disciplines of science (Schneider 2015), including in the context of plant pathology (Madden et al. 2015). In this article, we extend this discussion to emphasize the importance of considering multiple types of incorrect conclusions that can be reached in hypothesis testing. The two most commonly considered incorrect conclusions are known as Type I and Type II errors. Type I errors occur when the null hypothesis is rejected wrongly (e.g., that two treatment means are equal) in favor of an alternative hypothesis (e.g., that the two means are different). Type I errors are probably most familiar to researchers and much effort is expended to ensure that Type I errors are minimized. Type II errors also are common in hypothesis testing. These errors result from accepting the null hypothesis when in fact a treatment effect exists.

Studies that report no statistical differences between or among treatments may suffer from Type II errors because there are two possible interpretations when no significant difference is found. The treatments being evaluated indeed may not have a significant effect, and a correct conclusion was reached. Alternatively, a treatment effect may exist but the study wrongly concluded there was not a significant effect because of inadequate statistical power to detect differences among treatments (Fig. 1).

†Corresponding author: D. H. Gent; E-mail: dave.gent@ars.usda.gov

The use of trade, firm, or corporation names in this publication is for the information and convenience of the reader. Such use does not constitute an official endorsement or approval by the United States Department of Agriculture or the Agricultural Research Service of any product or service to the exclusion of others that may be suitable.

This article is in the public domain and not copyrightable. It may be freely reprinted with customary crediting of the source. The American Phytopathological Society, 2018.
Contemporary null hypothesis significance testing often neglects the importance of minimizing Type II errors. In this article, our goal is to raise awareness of the importance of power analysis in research planning and reporting of results. Experimental design and power analysis is a vast topic with numerous considerations that this paper cannot fully address. Therefore, we have structured this article as an introduction to the conceptual basis of statistical power and the critical importance of understanding power in designing experiments and appropriately interpreting results. The intended audience is the general reader of this journal for which we assume only an introductory knowledge of statistics. Firstly, we describe the concept of statistical power and why formalized power analysis is a necessary component of research planning. Secondly, we present important concepts of effect size and sample size necessary for calculation of power. We then discuss approaches to power calculation, with special emphasis on modern statistical analyses using mixed models. With this background, we present readers with practical advice on how to improve statistical power and demonstrate these concepts with two examples. We end with a final discussion and recommendations for the readership and Editorial Board of Phytopathology.

\section{THE IMPORTANCE OF POWER ANALYSIS}

Statistical power of a study is the conditional probability of rejecting the null hypothesis when the alternative is true (Murphy et al. 2014). This probability is conditioned on an effect size, a threshold probability for Type I error ( $\alpha$ level), and sample size. Given a Type II error of $\beta$, power is the complement, $1-\beta$. Stated plainly, power is the probability that a specified effect size can be distinguished from random variation of the variable being measured. An effect size is a standardized difference between treatments or the strength of a relationship among variables (Bausell and $\mathrm{Li}$ 2006). This may be the difference in means of two treatments, the correlation of two variables, or many other possible expressions of 
standardized differences indexed in units appropriate to specific data (Cohen 1988). A study is said to be underpowered if the study lacks sufficient power to detect reliably an effect size of interest.

Insufficient statistical power is prevalent in scientific literature (Ioannidis 2005; Levine et al. 2009; Sedlmeier and Gigerenzer 1989). When power is calculated retrospectively, many published studies reporting nonsignificant results are found to be severely underpowered and may only have been able to detect large treatment effects (Cohen 1962; Moher et al. 1994). Tressoldi et al. (2013) found fewer than $3 \%$ of articles in the journals Science and Nature even stated if a power analysis was conducted.

Conduct of underpowered research raises ethical questions (Halpern et al. 2002). With underpowered experiments, multiple studies may be required to detect an adverse treatment effect, involving greater risk of more study participants and inefficient use of resources (Button et al. 2013). When the costs and potential impacts of Type II errors are large, it is also reasonable to require a researcher or industry to demonstrate, with a high degree of certainty, that there is no adverse effect of a treatment before recommending a practice or permitting an activity (Dayton 1998; Peterman 1990). For instance, the U.S. National Institutes of Health (NIH) and U.S. Food and Drug Administration (FDA) routinely require justification of sample sizes, including power calculation, and discussion of effect size in relation to potential clinical impact in funding requests and experimental protocols (NIH transparency in reporting website; Redbook 2000). While this level of rigor may not be required in many plant pathology studies, formalized power analysis can be considered a best practice in science (Gbur et al. 2012). Ensuring adequate power to detect small yet biologically relevant effects is especially critical when treatments can reduce yield or crop quality (e.g., as a result of changes in cultural practices or using fewer fungicide applications).

Given a primary focus on statistical significance in scientific publishing it is surprising that approaches and practices that improve power, and therefore the probability of obtaining statistically significant results, are underutilized (Bausell and Li 2006). A fundamental problem in experimental design is that in the absence of statistical guidance heuristics are used to make decisions (Slovic et al. 1974). A heuristic decision can lead to serious biases and overconfidence in judgments, including individuals with formal training in statistics, probability, and experimental design. Tversky and Kahneman (1971) found that researchers in the social sciences often relied on educated intuition when making decisions on sample sizes and other critical aspects of experimental design, resulting in unwarranted confidence in small sample sizes and misplaced trust in trends in the earliest data points collected. They concluded that reliance on formal statistical procedures is essential for sound experimental design and analysis to keep cognitive biases in check. Formal consideration of statistical power of an experiment should therefore be a routine and indispensable component of experimental design before data collection and analysis. Below, we present basic concepts and considerations for power analysis.

\section{EFFECT SIZE AND CALCULATION OF POWER}

Determination of appropriate sample sizes and calculation of power requires an estimate of an effect size. As noted previously, an

True status of treatments effect

\begin{tabular}{|c|c|c|c|}
\hline \multirow{3}{*}{ Conclusion from study } & \multirow[b]{2}{*}{ No treatment effect } & No treatment effect & Treatment effect \\
\hline & & $\begin{array}{l}\text { Correct conclusion } \\
(P=1-\alpha)\end{array}$ & $\begin{array}{l}\text { Type II error } \\
(P=\beta)\end{array}$ \\
\hline & Treatment effect & $\begin{array}{l}\text { Type I error } \\
(P=\alpha)\end{array}$ & $\begin{array}{l}\text { Correct conclusion } \\
(P=1-\beta)\end{array}$ \\
\hline
\end{tabular}

Fig. 1. Possible outcomes from hypothesis testing studies. The shaded box represents statistical power. effect size is a standardized difference in means between groups or treatments in a study or the strength of the relationship between variables (Cohen 1988). Any parameter or combinations of parameters may be considered an effect size and each statistical test has its own effect size, ranging upward from 0 (Cohen 1988). In the Neyman-Pearson formulation of a hypothesis test the effect size is posited as an exact size of the effect an experiment is designed to detect (Neyman and Pearson $(1928 ; 1933)$. Conversely, in Fisher's formulation the effect size is zero (Fisher 1925).

Expression of an effect size in standardized form removes the original scale used when measuring a variable, allowing comparison of the strength of a treatment when measured with disparate scales. In a simple case with two independent treatments, the standardized effect size, $d$, for two treatments with expected means $\mu_{1}$ and $\mu_{2}$, and pooled standard deviation $\sigma$ is

$$
d=\frac{\mu_{1}-\mu_{2}}{\sigma}
$$

This effect size expresses the mean difference between two populations as a percentage of the common standard deviation. For the example in equation 1 with two independent means, Cohen (1988), in the context of social sciences, suggested that $0.2,0.5$, and 0.8 can be considered small, medium, and large effect sizes, respectively. An effect size of $d=0.5$ is equal to a difference in means of half a standard deviation. Effect size equivalents of $d$ are available for other common statistics, such as the coefficient of determination $\left(R^{2}\right)$. Cohen $(1988 ; 1992)$ gives tables of effect size indexes for several common statistical tests and operational definitions of what may be considered small, medium, or large effects. In plant pathology, operational definitions of small, medium, and large effect sizes have not been proposed and will vary depending on the specific pathosystem and research question.

A hypothesized effect size may be determined from a preliminary experiment, scientific literature, or judgment and experience of the researcher. In the absence of other evidence, Cohen (1988) suggested choosing a medium effect size, with the value of "medium" being context-specific. An alternative is to specify an effect size that represents the smallest effect of biological relevance. For instance, when screening fungicides one might have a sense of effect sizes that would be required to be useful for a given disease. If a standard fungicide provides, for example, $60 \%$ disease control, identifying a new fungicide with a similar mode of action that provides $65 \%$ disease control may not be meaningful, whereas $80 \%$ disease control could be meaningful. A power analysis is then aimed at detecting effect sizes of biologically meaningful differences (e.g., 20\% improvement in disease control), as we will discuss and illustrate later. It is also advisable to consider a series of effect sizes and conduct a sensitivity analysis to understand how statistical power may vary given a range of treatment differences.

Power and sample size analyses also are influenced by variability in measurement of the response variable. In the simple example in equation 1, this variability is expressed as the standard deviation $\sigma$, which is incorporated into the effect size calculation. With other statistical tests, explicit measures of variance may be expressed separately from expected differences in treatment means (Gbur et al. 2012). Measurement variability is the sum of biological, environmental, and other experimental sources influencing the variable being measured. For example, this may be due to patchy inoculum distribution in a field plot, temperature gradients across a greenhouse, disease assessment errors, and similar factors. As with hypothesized effect sizes, measurement variability can be estimated from preliminary experiments, related literature, and/or experience.

With an effect size(s) and measure of variability, determination of an appropriate sample size for a prespecified $\alpha$ and desired power is relatively straightforward for simple experimental designs with fixed effects and a normally distributed response variable. The choice of the equation (or reference table) to use to calculate power 
or sample size depends on the study design and statistical test; simple equations do not exist for mixed models with non-normal response variables. Therefore, the intended statistical model for analysis needs to be identified in the planning stages of an experiment. In the simple case of two sample means, a power calculation is merely the probability $(p)$ of obtaining a $z$-score $(z)$ of at least the hypothesized $t$ value $\left(t_{\text {hyp }}\right)$ - a critical value of $t\left(t_{c v}\right)$ necessary to achieve statistical significance at some specified level of $\alpha$. More formally, the equation for power in this example is given as

$$
\text { Power }=p\left(z \leq \frac{t_{h y p}-t_{c v}}{\sqrt{1+\frac{t_{c v}^{2}}{2 \mathrm{df}_{t}}}}\right)
$$

Conceptually in this example, power is the probability of obtaining a $t$ value $\left(t_{\text {hyp }}\right)$ that is greater than the critical value of $t\left(t_{c v}\right)$ needed for statistical significance to be obtained. Equation 2 expresses this probability based on a $z$-score from a standard normal distribution. The denominator is a correction factor to account for the skewed distribution of $t_{h y p}$ with small sample sizes (Hays 1973), where $\mathrm{df}_{t}$ is the degrees of freedom for $t_{c v}$. From the simple example in equation 2 , we see that power is a function of sample size and effect size (implicit in the calculation of $t_{\text {hyp }}$ and $\left.t_{c v}\right)$, and the threshold probability level for the statistical test (determined by the stringency of the $z$-score). When three of these four factors are known, the other factor can be calculated.

\section{THE APPROPRIATE LEVEL OF POWER}

The appropriate level of power depends on the impact of Type I versus Type II errors. Power often is set at 0.8 by convention (Cohen 1988), although power of 0.9 might be more appropriate when potential adverse treatment effects are important (e.g., in clinical trials, when evaluating treatments that might reduce yield of a high value crop, or if serious environmental hazards exists). Holding all other factors constant, a stringent $\alpha$ threshold reduces statistical power. It is instructive to consider explicitly the tradeoffs in experimental errors from varying combinations of $\alpha$ and $\beta$. When $\alpha$ is set nominally at 0.05 and $\beta$ at 0.2 (power $=0.8$ ), then an investigator is structuring tests assuming Type I errors are four times more important than Type II errors (i.e., $\beta / \alpha=0.2 / 0.05$ ). With $\alpha=$ 0.01 and power $=0.8$, Type I errors are controlled at 20 times the rate of Type II errors. Both ratios are arguably disproportionately skewed toward minimizing Type I errors for many studies in a plant pathology context, such as fungicide efficacy studies or cultivars trials. Explicit consideration of costs of false negative and false positive errors is routine in decision theory (Hughes and Madden 2003), and the same concepts are worthwhile to consider in experimental design when selecting appropriate ratios of $\alpha$ and $\beta$ (Cascio and Zedeck 1983). Madden et al. (2015) also discussed situations where values of $\alpha$ can be relaxed, such as in exploratory or preliminary experiments. While there is not a single appropriate level of power, studies with power less than 0.5 generally should not be conducted because there is less than a $50 \%$ probability of not detecting the hypothesized effect.

\section{PRACTICAL STEPS TO IMPROVE STATISTICAL POWER}

Power tends to be low when sample sizes, $\alpha$ level, and effect sizes are small. Researchers typically have some control over some of these factors, but often at the expense of increasing the cost of a study, modifying experimental designs and protocols, or changing measurement techniques. In this section, practical steps to improve statistical power are reviewed and explained. Other strategies that would be less common in plant pathology studies are presented by
Bausell and Li (2006) and Browner et al. (2001). Although the calculation of power differs, the strategies presented here apply both to fixed effect and mixed effect models.

Increase sample sizes. Increasing sample sizes invariably increases power. This is the most direct means to improve power but often the most expensive (Cohen 1988). A simple illustration of the effect of sample size is presented in Table 1 for a two-sample $t$ test for equal means (assuming equal variance). Independent of the value of $\alpha$ or the effect size, power increases with sample size. Given this, a further motivation for conducting a power analysis in the early planning stages of an experiment is to ensure that adequate resources are available to ensure a study has at least a plausible chance of being successful. When sample sizes are known to be inadequate due to factors outside of the control of a researcher, and results are nonsignificant, reporting power of the experiment is especially important (Levine et al. 2009).

There are, of course, trade-offs in what sample sizes are feasible and also ensure adequate power. Where fundamental limitations exist to what sample sizes can be used or processed in a given study, power calculations provide guidance on what effect size may be reliably detected within the constraints of one's budget, experimental design, and measurement precision (Quinn and Keough 2002). Tradeoffs in power with different experimental designs (e.g., single factor versus split-plot) can also be explored to select designs with the greatest chance of detecting relevant treatment effects (Gbur et al. 2012).

Often, power for an individual study may be unacceptably low (for example, see Figure 2A of Madden and Paul 2011) given resource limitations. Sufficient growth chamber space, fields, time to conduct sensitive measurements, or other resources may not be available to conduct a study with the number of samples needed to achieve a reasonable level of power to detect a small effect size. In such instances, replication of experiments in multiple locations or over time and aggregation of data (meta-analysis) can improve power substantially (Madden and Paul 2011). Multilocation experiments are common in agricultural research and clinical trials (Littell et al. 2006), and the statistical issues are the same as studies conducted in multiple runs over time (Gbur et al. 2012). Multilocation experiments might be especially attractive when logistical issues constrain the size of individual studies but small effect sizes are hypothesized. A good example of the value of this approach is found in Kandel et al. (2016).

Choose a less stringent alpha level. An $\alpha$ level of 0.05 is widely considered a critical threshold in scientific literature for historical reasons, although the stricture of this threshold versus a higher level reduces the likelihood of obtaining statistical significance and thus power. When sample size, experimental procedures, and effect size are fixed, adjustment of $\alpha$ may be the only way to increase power. Table 1 demonstrates this idea with a simple

TABLE 1. Influence of sample size, effect size, and significance level on the statistical power of a two-sample $t$ test for equal means

\begin{tabular}{lcccccccc}
\hline & \multicolumn{3}{c}{ Effect size $=0.5^{\mathrm{a}}$} & & \multicolumn{3}{c}{ Effect size $=2^{\mathrm{a}}$} \\
\cline { 2 - 4 } \cline { 7 - 8 }$N$ per group & $\alpha=0.01$ & $\alpha=0.05$ & $\alpha=0.1$ & & $\alpha=0.01$ & $\alpha=0.05$ & $\alpha=0.1$ \\
\hline 4 & 0.02 & 0.09 & 0.17 & & 0.31 & 0.66 & 0.80 \\
5 & 0.03 & 0.11 & 0.19 & & 0.48 & 0.79 & 0.89 \\
6 & 0.03 & 0.12 & 0.21 & & 0.62 & 0.88 & 0.94 \\
7 & 0.04 & 0.14 & 0.23 & & 0.74 & 0.93 & 0.97 \\
8 & 0.05 & 0.15 & 0.25 & & 0.83 & 0.96 & 0.98 \\
9 & 0.05 & 0.17 & 0.27 & & 0.89 & 0.98 & 0.99 \\
10 & 0.08 & 0.19 & 0.29 & & $>0.99$ & 0.99 & $>0.99$ \\
20 & 0.14 & 0.34 & 0.46 & & $>0.99$ & $>0.99$ & $>0.99$ \\
40 & 0.35 & 0.60 & 0.72 & & $>0.99$ & $>0.99$ & $>0.99$ \\
80 & 0.71 & 0.88 & 0.93 & & $>0.99$ & $>0.99$ & $>0.99$ \\
\hline
\end{tabular}

${ }^{a}$ For a two-sample $t$ test with equal variance among samples, an effect size of 0.5 is equivalent to a difference in means equal to one half of one standard deviation. An effect size of 2 is a difference in means equal to two standard deviations. 
experimental design. Sample sizes must become exceptionally large (i.e., much larger than typical studies published in Phytopathology) when stringent values of $\alpha$ are desired, even with relatively large effect sizes. Existing standards and editorial board policies may make it difficult to justify deviation upward from an $\alpha=0.05$, although setting $\alpha$ lower and leaving $\beta$ unknown generally is not questioned. In other situations, such as exploratory research, values of $\alpha$ greater than 0.05 often are acceptable (Madden et al. 2015).

Cascio and Zedeck (1983) present a simple decision-theoretic formula for choosing a sensible value of $\alpha$ based on an expected probability that a null hypothesis is incorrect, the desired power, and the relative consequence of Type I versus Type II errors. The relative seriousness of mistakenly rejecting an alternative hypothesis when it is actually true can be expressed as

$$
\text { Relative seriousness }=\frac{p\left(\mathrm{H}_{1}\right) \beta}{\left[1-p\left(\mathrm{H}_{1}\right) \alpha\right]}
$$

where $\alpha$ and $\beta$ are the probability of Type I and Type II errors, and $p$ $\left(\mathrm{H}_{1}\right)$ is the probability of the alternative hypothesis being true. More concretely, suppose one is interested in determining whether two cultivars, with similar agronomic and quality characteristics, differ in their susceptibility to a disease. In the planning stages of an experiment, one follows convention and designs an experiment with a sample size sufficiently large to obtain power $=0.8$ based on the hypothesized effect size with $\alpha$ nominally set to 0.05 . From a previous study, we expect that there is 0.7 probability that the cultivars do indeed differ in disease susceptibility. According to equation 3 , by uncritically setting $\beta=0.2$ and $\alpha=0.05$ we are implicitly assuming the relative seriousness of Type I versus Type II errors is $(0.7 \times 0.2) /(0.3 \times 0.05)=9.33$. Relaxing $\alpha$ to 0.1 changes the ratio to 4.67. The appropriate ratio to accept will depend on the consequences of each type of error, but in this example selecting nominal values of $\beta$ and $\alpha$ leads to an overly conservative view of the importance of Type I errors given our expectation $\left(p\left(\mathrm{H}_{1}\right)=0.7\right)$ that the cultivars do vary in disease susceptibility.

When there is very strong evidence that the null hypothesis is true (e.g., $\left.p\left(\mathrm{H}_{1}\right)>0.9\right)$ the relative seriousness of errors becomes highly asymmetric with $\alpha$ set to 0.05 or less (Murphy et al. 2014). When the relative seriousness of Type I and Type II errors can be expressed concretely, equation 3 can easily be rearranged to solve for the level of $\alpha$ that appropriately balances Type I and Type II errors. Explicit consideration of errors often will lead to relaxing $\alpha$ thresholds.

In many studies, when the direction of a treatment response is known with near certainty (e.g., a given fungicide reduces disease) then use of a one-tailed test is appropriate and approximately increases $\alpha$ to 0.10 . There is a caution with use of one-tailed tests, though. Directional tests cannot reject a null hypothesis when the direction of a treatment effect is opposite to that predicted (e.g., a fungicide increases disease). Thus, by definition, directional tests have no power to detect these effects (Cohen 1988).

TABLE 2. Power of a one-way analysis of variance with two treatment groups, an effect size 0.5 , and $N=20$ individuals per group when a covariate of varying magnitude (expressed as $r$ ) is included in the analysis ${ }^{a}$

\begin{tabular}{lcc}
\hline$r$ & Adjusted effect size & Power \\
\hline$\ldots$ & 0.50 & 0.58 \\
0.1 & 0.50 & 0.58 \\
0.2 & 0.51 & 0.60 \\
0.3 & 0.53 & 0.63 \\
0.4 & 0.56 & 0.68 \\
0.5 & 0.60 & 0.74 \\
0.6 & 0.67 & 0.83 \\
0.7 & 0.80 & 0.93 \\
0.8 & 1.12 & $>0.99$ \\
\hline
\end{tabular}

a In this example, the effect size is expressed as $f$, the standard deviation of the group mean from the mean of the combined groups, standardized by the common standard deviation of the groups (Cohen 1988).
One-tailed tests have limitations when a large numbers of treatments are evaluated and corrections for multiple comparison tests are needed to control Type I errors. Corrections for multiple comparisons may reduce power substantially as compared with evaluations of smaller numbers of treatments or preplanned comparison of treatments, similar to the impact of reducing $\alpha$.

Increase the hypothesized effect size. Effect size is a crucial parameter in power analysis, but perhaps the most difficult to set when designing an experiment. Nonetheless, increasing the minimum detectable effect size, while holding $\alpha$, sample size, and variance constant, will increase power. As noted previously, effect sizes should reflect the minimum, biologically relevant difference in a parameter of interest; setting effect sizes smaller will decrease power. For example, an experiment designed to detect a $10 \%$ yield increase will have greater power than an identical experiment desiring to detect a 5\% yield increase. Relying again on the example in Table 1, note the substantial improvement in power (and commensurate reduction in sample size) as effect size increases. Thus, careful consideration of the minimum, biologically relevant effect can improve power.

This approach is problematic when the true effect size is unknown or is small, and may not be practical in many situations with biologically important and relevant questions. In some studies though, treatments can be evaluated at a time period when effect sizes are expected to be maximal. For instance, in studies screening different fungicides, increasing doses of fungicides or considering the timing of studies to correspond with periods when treatment effects are maximized can increase the effect size and power.

Consider as few treatment groups as possible. In factorial designs, power to detect interactions of factors can be particularly low because effect sizes for interactions often are smaller than main factors and because main effects have more degrees of freedom (Murphy et al. 2014). Reallocating the total number of subjects or plots available to a larger number of groups will reduce the power of a study, sometimes dramatically and unacceptably, even when holding an effect size constant (Bradley and Russell 1998). When biological questions of interest necessitate complex designs, sample sizes may need to become quite large to ensure adequate power to detect interactions. In these instances, strategies such as obtaining multiple measurements per experimental unit over time (repeatedmeasures designs) may allow one to collect a large amount of data from a small number of experimental units without sacrificing power (Murphy et al. 2014).

Choose continuous rather than categorical dependent variables. Use of categorical and ordinal variables is common in plant pathology studies because of the difficulty of rating some symptoms (Shah and Madden 2004). For a fixed sample size, statistical tests based on the proportion of individuals in a category have less power than tests based on continuous variables, and dichotomization of data that could be analyzed on a continuous scale is strongly discouraged (Beckstead 2012; Beckstead and Beckie 2011; Dawson and Weiss 2013). When ordinal variables are used for convenience or necessity, adjustments in experimental procedures may need to be made to compensate for the loss of power.

Utilize covariates and consider blocking to control variables that may influence treatment effects. Controlling for variability in a response variable by imposing a blocking structure and inclusion of relevant covariates increases estimated effect sizes and power. The increase in power due to inclusion of a covariate is directly related to the strength of the correlation of a covariate and dependent variable, due to reducing unexplained residual variance (Cohen 1988). This concept is demonstrated in Table 2, assuming an effect size of 0.5 , sample size of 20 individuals per group, and $\alpha=$ 0.05 . Including a covariate in the analysis increases power substantially in proportion to the strength of the correlation with the response variable. While inclusion of covariates is often advocated for improving estimation of a parameter, this is also a useful data 
analytic device that should be employed when possible (Cohen 1988).

Improve reliability and sensitivity of measurements. Measurement errors attenuate (shrink) observed effect sizes (Cohen 1988). Notice in Equation 1, that a reduction of the standard deviation by half doubles the effect size. Because large effect sizes increase power, power can be improved by modifying measurement approaches to increase the strength of the correlation between a response variable and an independent variable. This could be as simple as making two measurements per experimental unit instead of one. In studies where disease assessments are involved, selecting a measure of disease intensity and spatial scale for sampling (e.g., leaflet, leaf, plant) that maximizes the reliability of the assessments and differences among treatments of interest should be considered. Training of raters (Nutter and Schultz 1995) and use of image analysis software (Lamari 2002; Pethybridge and Nelson 2015) can also improve reliability.

In instances where multiple, interrelated response variables could potentially be measured, measurement of the variable that is most sensitive to change and that can be measured most reliably will improve power. For instance, where a genetic locus confers partial resistance to a disease, pleiotrophic effects on disease phenotype can be manifested as a lengthening of the latent period, increased sporulation of the pathogen, and enhanced infection efficiency (Wolfenbarger et al. 2016). Power will be improved by focusing efforts on measuring the variable with the largest effect size and smallest variance.

\section{POWER CALCULATIONS}

Power and sample size tables are included routinely in introductory statistics textbooks for simple statistical tests, or can be calculated with most statistical analysis programs. Because of the ease of calculating power with modern software and computing capabilities, some authors recommend using exact formulas even for simple experimental designs and statistics (Liu 2014). However, others (Bausell and Li 2006) suggest that exact formulas for determining sampling size are unnecessary given that the calculations are only estimates based on assumptions of the effect size. Consultation of tables with estimates of power or sample sizes for a given effect size are generally adequate when planning studies with simple experimental designs.

Methods to determine power and sample sizes exactly do not exist for all statistical tests (Eng 2004). Mixed effect models are becoming standard in plant pathology and other disciplines, and power analysis with these models is critically different than conventional models that assume only fixed effects. Power analysis in a mixed framework is more intensive computationally and complicated because of the complex covariance structure of mixed effect models, and possibly non-normal data. Bausell and $\mathrm{Li}$ (2006) suggested that when power analytic approaches have not been developed for a specific statistical procedure, that researchers use a more conventional analytic approach or model power based upon the closest simple analog, and explicitly state the basis of the calculations done. Other authors contend that power analysis in modern statistical models depends absolutely on using generalized linear mixed model theory and methods (Stroup 2016). Although more computationally demanding, we agree with Stroup (2016) that methods appropriate for non-normal data and mixed models should be employed because of the dependence of power on the distribution of the response variable and variance structure. Methods based on an analytical approximation of a probability distribution exist when an exemplar data set can be generated from a preliminary experiment or other sources; examples of this approach are provided by Littell et al. (2006) and Gbur et al. (2012). Liu (2014) presents a comprehensive treatment of power analysis with random effects and multilevel models. As with power analysis with fixed effect models, one needs to specify a minimum treatment effect size, the magnitude of variation and correlation in the data, and the structure of the proposed experimental design. The probability distribution of the response variable also needs to be specified with generalized mixed effect models.

Alternatively, and more commonly, power is assessed in mixed effect models by simulation (Gbur et al. 2012; Green and MacLeod 2016; Johnson et al. 2015; Kain et al. 2015; Littell et al. 2006). In this approach, a random number generator is used to create hundreds or thousands of independent data sets that match the experimental design, treatment effects, probability distribution of the response variable, and estimated values for variance and correlation parameters (Gbur et al. 2012). Each of the simulated data sets is analyzed in the appropriate generalized linear mixed model and the proportion of simulated experiments where statistical significance is achieved is taken as the estimated power. With more complex experimental designs and mixed models more parameters need to be estimated, which can be difficult in areas of new research where preliminary data and literature do not exist. Gbur et al. (2012) advocate using a two-step approach to power analysis with mixed models, where power of a study is evaluating using a probability distribution method with an exemplar data set and the results then verified via simulation, especially with generalized linear mixed models.

Worked examples. To demonstrate these concepts, two examples are presented for common experimental designs and analysis problems encountered in plant pathology. We emphasize that in each case the value of power analysis is early in the planning phases of a study and should involve consultation with a statistician.

Example 1. Pearson's correlation coefficient. Power curves for the Pearson correlation coefficient, $r$, are presented in Figure 2. The Pearson correlation coefficient is an index that describes the strength of a linear relationship between pairs of two standardized variables, and when used as a descriptive measure, $r$ is an effect size (Cohen 1988). Casual inspection of the power curves indicates that power and sample size ( $n$ pairs of two variables) are largely influenced by $r$. For illustrative purposes, consider that power $=0.8$ is desired. To achieve power $=0.8, n$ increases from $7,17,44$, to 191 as $r$ decreases from 0.8, 0.6, 0.4, to 0.2 at $\alpha=0.05$ (Fig. 2A). Samples sizes become especially large when $\alpha$ is set to 0.01 , being $11,25,65$, and 284 for the same values of $r$. Thus, relatively large sample sizes are necessary when detecting effect sizes of less than 0.6 , and examples in APS journals of underpowered studies for detecting these effect sizes are common (e.g., Gent et al. [2004] to point out but one example).

When resource limitations and logistical issues preclude use of an adequately large sample size, increasing the strength of the relationship between the variables will increase power for a given $n$. Suppose a sample size of $n=30$ was the largest sample possible for a study, then an observed effect size $r=0.47$ would be necessary to ensure power of 0.8 . If observed $r$ was less than 0.47 due to measurement errors, efforts could be made to improve the reliability of measurements (with different methods or selection of more sensitive response variables). In an instance where the true correlation of two variables was, say, 0.5 and the actual measurement of the variables correlated at 0.7 due to the reliability of the measurement system, the observed (fallible) correlation would be $0.5 \times 0.7 \times 0.7=0.25$ and power of the experiment would be 0.28 . However, use of a more reliable measurement instrument (e.g., image analysis instead of visual assessment of disease severity) that improved the correlation between the observed and true correlation to 0.97 would increase power to 0.8 . Relaxing $\alpha$ to 0.1 (or considering only a one-sided correlation a priori) would yield power of 0.40 with the original measurement systems, and power of 0.89 with the more precise measurement instrument. Repeating the experiment in time, with the same sample size, would increase power to 0.5 with the original measurements system and 0.98 with more precise measurements at $\alpha=0.05$. Both repeating the experiment and accepting $\alpha=0.1$, power increases to 0.63 with the original 
measurement instrument and 0.99 with the more precise measurements. With whatever course of action is taken, reporting of results should state explicitly the limits of the study and the potential for a true effect size smaller than what could be detected reliably within the experimental constraints.

Example 2. Randomized complete block design with a binomial response variable. As a second illustration, examples 7.3 and 7.4 of Gbur et al. (2012) are considered and expanded on in a plant pathology context. Suppose one is planning a simple field experiment that will evaluate the impact of nitrogen fertilization rate on the incidence of a disease. To make this example more concrete, consider specifically the disease powdery mildew on hop, where infection of the harvested cones is the primary economic concern for disease management (Mahaffee et al. 2009). As is typical with many powdery mildew diseases, we expect that large doses of nitrogen fertilizer may increase levels of the disease and an experiment is planned to test this hypothesis. Based on preliminary data and experience with this pathosystem, we anticipate that the standard fertilization practices (the control in this experiment) will result in $80 \%$ of the cones becoming diseased by harvest, whereas reducing fertilization would reduce disease by some unknown amount. For the disease reduction to be relevant biologically and to producers, we establish that disease levels should be reduced such that no more than $60 \%$ of the cones are diseased. This becomes the minimum effect size for the power analysis.
Given inherent spatial variability in field studies with this crop and disease, a randomized blocking structure is deemed appropriate. Since the response variable of interest is disease incidence, a generalized linear mixed model is needed to account for the binomial distribution of the response variable, with random factors for block and treatment-block interaction to control for probable over dispersion of the data (Gbur et al. 2012). From preliminary experiments, we estimate the variance component for the block factor to be 0.03 and for the treatment-block interaction to be 0.24 .

Assessing cones for this disease is time consuming, and we desire to evaluate as few cones as feasible while ensuring adequate statistical power. How many replicate blocks and cones per plot are needed to detect the minimum, nontrivial effect size given the experimental design and variability among blocks and among treatments from block to block? Following the methods of Gbur et al. (2012) and Littell et al. (2006), an exemplary data set is constructed, considering four to eight replicate blocks with either 75 or 150 cones rated per plot. This constructed data set is then analyzed using a generalized linear mixed model to obtain the $F$ value and numerator and denominator degrees of freedom from a least square means comparisons needed to calculate the approximate power (Table 3).

Power invariably increases with increasing sample size, based on either the number of blocks or the number of cones rated for disease within each plot. As expected, power also increases when $\alpha$ is relaxed from 0.05 to 0.1 (Table 3 ). The approximate power of the
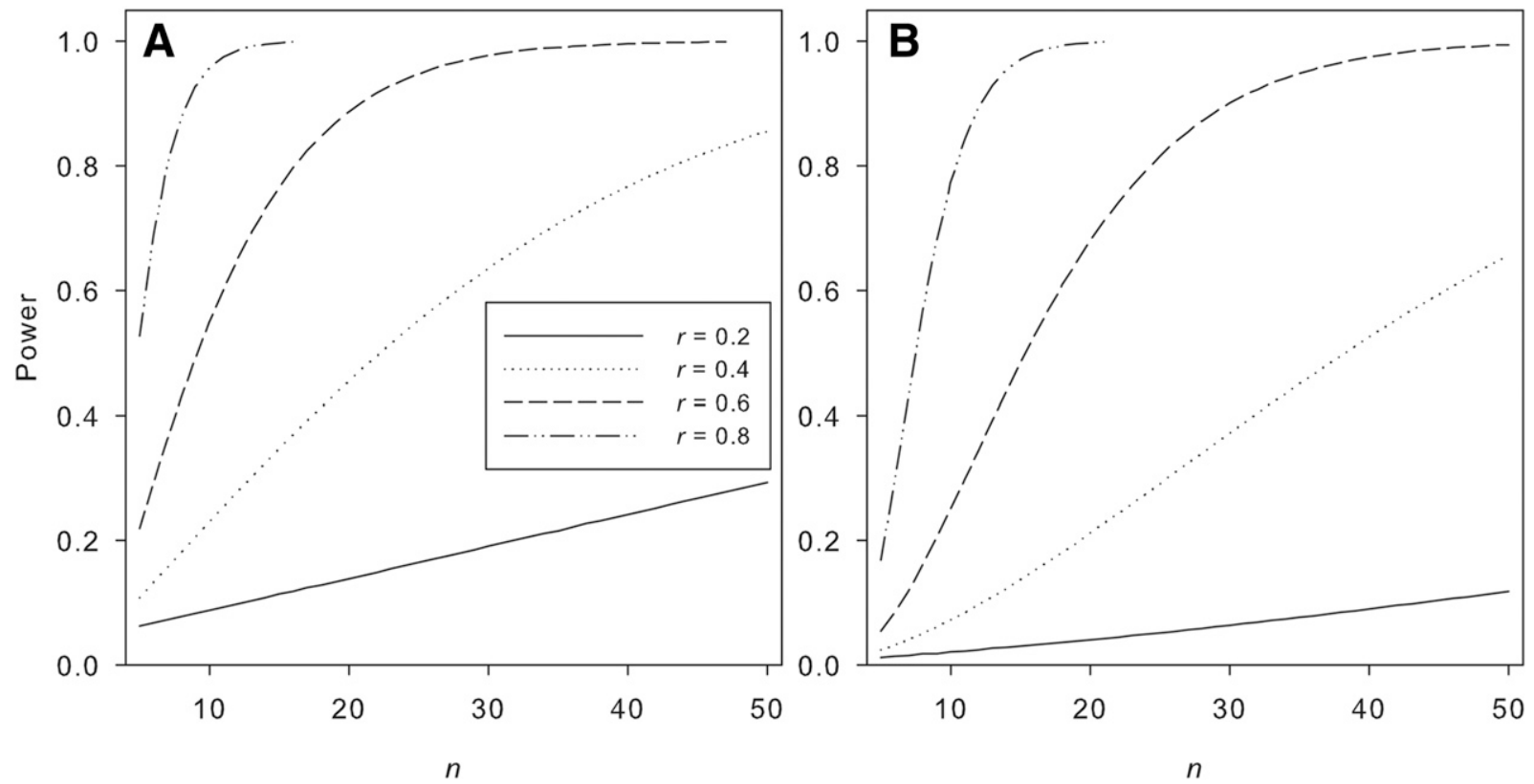

Fig. 2. Power and sample size relationships for the correlation between two variables (a random variable $Y$ and a fixed variable $X$ ) with correlation coefficients of $0.2,0.4,0.6$, and 0.8. Power curves are shown for $\mathbf{A}, \alpha=0.05$ and $\mathbf{B}, \alpha=0.01$.

TABLE 3. Approximate power needed to detect treatment differences and the time to rate disease in an exemplary data set with various combinations of blocks, samples per plots, and significance thresholds ${ }^{\mathrm{a}}$

\begin{tabular}{|c|c|c|c|c|c|c|c|c|}
\hline \multirow[b]{2}{*}{ Number of blocks } & \multicolumn{4}{|c|}{$n=75$ per plot } & \multicolumn{4}{|c|}{$n=150$ per plot } \\
\hline & $\alpha=0.05$ & $\alpha=0.1$ & Total $n$ & Approximate hours to rate ${ }^{b}$ & $\alpha=0.05$ & $\alpha=0.1$ & Total $n$ & Approximate hours to rate \\
\hline 4 & 0.41 & 0.60 & 600 & 7.2 & 0.44 & 0.65 & 1,200 & 14.4 \\
\hline 5 & 0.56 & 0.74 & 750 & 9.0 & 0.61 & 0.78 & 1,500 & 18.0 \\
\hline 7 & 0.78 & 0.90 & 1,050 & 12.6 & 0.83 & 0.92 & 2,100 & 25.2 \\
\hline 8 & 0.85 & 0.94 & 1,200 & 14.4 & 0.89 & 0.96 & 2,400 & 28.8 \\
\hline
\end{tabular}

a Power was approximated using the probability distribution method (Gbur et al. 2012; Littell et al. 2006) with an exemplary data set for a randomized complete block design with two treatments, assuming analysis in a conditional generalized linear mixed model with a binomial distribution of the response variable. Disease incidence associated with the two treatments was estimated as 0.6 and 0.8 , block variance as 0.03 , and block-treatment variance as 0.24 based on a preliminary experiment.

b Time for disease rating was based on an estimated 6 hours to rate 1500 hop cones for the presence of powdery mildew when using three trained raters (Gent et al. 2007). 
experimental design depends not only on the total number of cones assessed, but also the combined effects of number of blocks and cones assessed within each plot. Again, nominally assuming power of at least 0.80 is desired, eight blocks would be required when 75 cones are assessed per plot, whereas seven blocks would be adequate when the number of cones assessed per plot is doubled to 150. In the first scenario, though, the experimental design minimizes the time and number of cones that need to be rated. Because our interest is specifically in whether reduced nitrogen fertilization decreases disease levels, a one-sided test may be appropriate. With $\alpha=0.1$, adequate power is obtained with six blocks with either 75 or 150 cones assessed per plot. In this example only two treatments were considered, but the approach can be extended to comparison of multiple treatments. The optimum number of experimental units and blocks to maintain a given power changes with the number of treatments evaluated (Bradley and Russell 1998). Depending on the time, costs, and logistical constraints associated with deploying treatments in a field, tradeoffs in possible experimental designs can be evaluated explicitly to select the most efficient design.

\section{DISCUSSION}

The emphasis on power analysis should be in the planning stages of an experiment, rather than after a study is conducted using observed effect sizes. The intended power of an experiment is based on assumptions of effect size and variance, and the actual power of an experiment may differ. Nonetheless, the value of power analysis is in formalizing hypotheses, making explicit the limitations of a study, and in executing experiments that are more likely to detect postulated, relevant, treatment effects. The experimental design of many studies is framed around exigencies of money, time, and access to samples, and power analysis has much to offer beyond simply a means to determine sample sizes. Power analysis is a step to explore the costs and limitations of competing approaches and to plan an efficient study within resource constraints and also understand the limits of a study for detecting various effect sizes. Perhaps unexpectedly, inadequately powered studies that rely on threshold $P$ values for declaring significance are also more prone to detection of inflated effect sizes (termed Type M errors) [reviewed in Ioannidis (2008)] and Type S errors (Gelman and Tuerlinckx 2000). Type M errors result when one has correctly rejected a null hypothesis of no treatment effect, but the magnitude of the estimated effect size is inflated. AType S error corresponds to wrongly identifying the sign of a comparison (Gelman and Tuerlinckx 2000). Type M errors are most common in initial studies with small sample sizes. The combination of publishing new findings where little prior data exists and the draw of reporting dramatically large effect sizes can lead to high impact journals generally being prone to publishing inflated effect sizes (Brembs et al. 2013; Siontis et al. 2011). A properly conducted power analysis minimizes the risk of these errors.

As we discussed, power analysis is complicated for more complex analyses that involve non-normal data or violate assumptions of convention statistical analyses. These kinds of analyses are becoming increasingly standard as the limitations of ANOVA become more evident (Stroup 2015). An important category of such analyses is with repeated measurements made on the same sampling unit over time, which require mixed models to account for serial correlation of residuals. The most efficient experimental design depends on expected effect sizes and relative costs for establishing a treatment group versus the measurement costs of the response variable (Bradley and Russell 1998; Kain et al. 2015). A complication that surfaces is that there is not a single, universal means to estimate the covariance matrix in a repeated measures analysis for determining power, and simplifying assumptions about correlation structure must be made (Liu 2014). In reality, the appropriate correlation structure that describes a data set generally is determined statistically during model fitting and complex correlation structures are common (Littell et al. 2006). This introduces another set of assumptions that must be made and parameters to be estimated, further taxing most biologists attempting to calculate power and properly design an experiment. Consultation with a capable statistician should be considered no less than compulsory for power analyses involving complex experiments.

As a final caution, we noted that it is possible to calculate power post hoc, using estimated variance from the study and a specified effect size (Quinn and Keough 2002). This approach generally is discouraged because the so-called observed power of a study is determined completely by the achieved $P$ value (Hoenig and Heisey 2001). In these cases, retrospective power analysis changes nothing in the interpretation of $P$. A better approach is to report on power analyses conducted in the planning stages of an experiment. For simple experimental designs, this should include the power obtained in the stated experimental design based on a specified minimum effect size, $\alpha$, number of tails of the test and variance(s) for the experimental design (Bausell and Li 2006).

Many interesting and important problems in plant pathology involve phenomena for which effect sizes are small, yet biologically important for understanding epidemic behavior and disease management. Power analysis in such instances is especially important as underpowered studies may be misleading if nonsignificant results are not interpreted carefully. With adequate statistical power, the cost of conducting an individual experiment likely will increase as sample sizes become larger, fewer treatments are evaluated, more precise measurements are taken, and more targeted studies are conducted (Cohen 1990). However, the conclusions of these studies will more often be correct and reproducible. Presentation of the results of power analyses, when possible, is an important aspect of communicating these conclusions.

\section{ACKNOWLEDGMENTS}

We thank G. Hughes and L. du Toit for their insightful comments and suggestions that improved this paper. Support was provided by the U.S. Department of Agriculture Current Research Information System project number 5358-21000-046-00D.

\section{LITERATURE CITED}

Bausell, R. B., and Li, Y.-F. 2006. Power Analysis for Experimental Research: A Practical Guide for the Biological, Medical, and Social Sciences. Cambridge University Press, Cambridge.

Beckstead, J. W. 2012. To be, or to be two, the question of dichotomizing variables. Int. J. Nurs. Stud. 49:635-636.

Beckstead, J. W., and Beckie, T. M. 2011. How much information can metabolic syndrome provide? An application of information theory. Med. Decis. Making 31:79-92.

Bradley, D. R., and Russell, R. R. 1998. Some cautions regarding statistical power in split-plot designs. Behav. Res. Methods Instrum. Comput. 30: 462-477.

Brembs, B., Button, K., and Munafò, M. 2013. Deep impact: Unintended consequences of journal rank. Front. Hum. Neurosci. 7:291.

Browner, W. S., Newman, T. B., Cummings, S. R., and Hulley, S. B. 2001. Estimating sample size and power. Pages 65-84 in: Designing Clinical Research: An Epidemiologic Approach, 2nd Ed. S. B. Hulley, S. R. Cummings, W. S. Browner, D. Grady, N. Hearst, and T. B. Newman, eds. Lippincott, Williams and Wilkins, Philadelphia, PA.

Button, K. S., Ioannidis, J. P. A., Mokrysz, C., Nosek, B. A., Flint, J., Robinson, E. S. J., and Munafò, M. R. 2013. Power failure: Why small sample size undermines the reliability of neuroscience. Nat. Rev. Neurosci. 14:365-376.

Cascio, W. F., and Zedeck, S. 1983. Open a new window in rational research planning: Adjust alpha to maximize statistical power. Person. Psychol. 36: 517-526.

Cohen, J. 1962. The statistical power of abnormal-social psychological research: A review. J. Abnorm. Soc. Psychol. 65:145-153.

Cohen, J. 1988. Statistical Power Analysis for the Behavioral Sciences, 2nd Ed. Erlbaum, Hillsdale, NJ.

Cohen, J. 1990. Things I have learned (so far). Am. Psychol. 45:1304-1312.

Cohen, J. 1992. A power primer. Psychol. Bull. 112:155-159.

Dawson, N. V., and Weiss, R. 2013. Dichotomizing continuous variables in statistical analysis: A practice to avoid. Med. Decis. Making 32:225-226. 
Dayton, P. K. 1998. Reversal of the burden of proof in fisheries management. Science 279:821-822.

Eng, J. 2004. Sample size estimation: A glimpse beyond simple formulas. Radiology 230:606-612.

Fisher, R. A. 1925. Statistical Methods for Research Workers, 1st Ed. Oliver and Boyd, London.

Gbur, E. E., Stroup, W. W., McCarter, K. S., Durham, S., Young, L. J., Christman, M., West, M., and Kramer, M. 2012. Analysis of Generalized Linear Mixed Models in the Agricultural and Natural Resource Sciences. American Society of Agronomy, Soil Science Society of America, Crop Science Society of America, Madison, WI.

Gelman, A., and Tuerlinckx, F. 2000. Type S error rates for classical and Bayesian single and multiple comparison procedures. Comput. Stat. 15:373-390.

Gent, D. H., Schwartz, H. F., and Khosla, R. 2004. Distribution and incidence of Iris yellow spot virus in Colorado and its relation to onion plant population and yield. Plant Dis. 88:446-452.

Gent, D. H., Turechek, W. W., and Mahaffee, W. F. 2007. Sequential sampling for estimation and classification of the incidence of hop powdery mildew II: Cone sampling. Plant Dis. 91:1013-1020.

Green, P., and MacLeod, C. J. 2016. SIMR: An R package for power analysis of generalized linear mixed models by simulation. Methods Ecol. Evol. 7:493-498.

Halpern, S. D., Karlawish, J. H. T., and Berlin, J. A. 2002. The continuing unethical conduct of underpowered clinical trials. JAMA 288:358-362.

Hays, W. B. 1973. Statistics for the Social Sciences. Holt, Rinehart, and Winston, New York.

Hoenig, J. M., and Heisey, D. M. 2001. The abuse of power: The pervasive fallacy of power calculations for data analysis. Am. Stat. 55:19-24.

Hughes, G., and Madden, L. V. 2003. Evaluating predictive models with application in regulatory policy for invasive weeds. Agric. Syst. 76:755-774.

Ioannidis, J. P. A. 2005. Why most published research findings are false. PLoS Med. 2:e124.

Ioannidis, J. P. A. 2008. Why most discovered true associations are inflated. Epidemiology 19:640-648.

Johnson, P. C. D., Barry, S. J. E., Ferguson, H. M., and Müller, P. 2015. Power analysis for generalized linear mixed models in ecology and evolution. Methods Ecol. Evol. 6:133-142.

Kain, M. P., Bolker, B. M., and McCoy, M. W. 2015. A practical guide and power analysis for GLMMs: Detecting among treatment variation in random effects. PeerJ 3:e1226.

Kandel, Y. R., Wise, K. A., Bradley, C. A., Chilvers, M. I., Tenuta, A. U., and Mueller, D. S. 2016. Fungicide and cultivar effects on sudden death syndrome and yield of soybean. Plant Dis. 100:1339-1350.

Lamari, L. 2002. ASSESS: Image Analysis Software for Plant Disease Quantification. American Phytopathological Society, St. Paul, MN.

Levine, D., Bankier, A. A., and Halpern, E. F. 2009. Submissions to Radiology: Our top 10 list of statistical errors. Radiology 253:288-290.

Littell, R. C., Milliken, G. A., Stroup, W. W., Wolfinger, R. D., and Schabenberger, O. 2006. SAS for Mixed Models. SAS Institute, Cary, NC.

Liu, X. S. 2014. Statistical Power Analysis for the Social and Behavioral Sciences. Routledge, New York.

Madden, L. V., and Paul, P. A. 2011. Meta-analysis for evidence synthesis in plant pathology: An overview. Phytopathology 101:16-30.
Madden, L. V., Shah, D. A., and Esker, P. D. 2015. Does the $P$ value have a future in plant pathology? Phytopathology 105:1400-1407.

Mahaffee, W. F., Engelhard, B., Gent, D. H., and Grove, G. G. 2009. Powdery mildew. Pages 25-31 in: Compendium of Hop Diseases and Pests. W. M. Mahaffee, S. J. Pethybridge, and D. H. Gent, eds. American Phytopathological Society, St. Paul, MN.

Moher, D., Dulberg, C. S., and Wells, G. A. 1994. Statistical power, sample size, and their reporting in randomized controlled trials. JAMA 272:122-124.

Murphy, K. R., Myors, B., and Wolach, A. 2014. Statistical Power Analysis, 4th Ed. Routledge, New York.

Neyman, J., and Pearson, E. S. 1928. On the use and interpretation of certain test criteria of statistical inference. Part I. Biometrika 20A:175-240.

Neyman, J., and Pearson, E. S. 1933. On the problem of the most efficient tests of statistical hypotheses. Trans. Royal Soc. Lond. Ser. A 231:289-337.

Nutter, F. W., Jr., and Schultz, P. M. 1995. Improving the accuracy and precision of disease assessments: Selection of methods and use of computeraided training programs. Can. J. Plant Pathol. 17:174-184.

Peterman, R. M. 1990. The importance of reporting statistical power: The forest decline acidic deposition example. Ecology 71:2024-2027.

Pethybridge, S. J., and Nelson, S. C. 2015. Leaf Doctor: A new portable application for quantifying plant disease severity. Plant Dis. 99:1310-1316.

Quinn, G. P., and Keough, M. J. 2002. Experimental Design and Data Analysis for Biologists. Cambridge University Press, United Kingdom.

Redbook. 2000. Toxicological Principles for the Safety Assessment of Food Ingredients. U.S. Food and Drug Administration. College Park, MD.

Schneider, J. W. 2015. Null hypothesis significance tests. A mix-up of two different theories: The basis for widespread confusion and numerous misinterpretations. Scientometrics 102:411-432.

Sedlmeier, P., and Gigerenzer, G. 1989. Do studies of statistical power have an effect on the power of studies? Psychol. Bull. 105:309-316.

Shah, D. A., and Madden, L. V. 2004. Nonparametric analysis of ordinal data in designed factorial experiments. Phytopathology 94:33-43.

Siontis, K. C., Evangelou, E., and Ioannidis, J. P. 2011. Magnitude of effects in clinical trials published in high-impact general medical journals. Int. J. Epidemiol. 40:1280-1291.

Slovic, P., Kunreuther, H. C., and White, G. 1974. Decision processes, rationality and adjustment to natural hazards. Pages 187-205 in: Natural Hazards: Local, National, Global. G. F. White, ed. Oxford University Press, New York.

Stroup, W. W. 2015. Rethinking the analysis of non-normal data in plant and soil science. Agron. J. 107:811-827.

Stroup, W. W. 2016. PROC GLIMMIX as a teaching and planning tool for experiment design. SAS Global Forum 2016. Paper 11663-2016.

Tressoldi, P. E., Giofré, D., Sella, F., and Cumming, G. 2013. High impact = high statistical standards? Not necessarily so. PLoS One 8:e56180.

Tversky, A., and Kahneman, D. 1971. Belief in the law of small numbers. Psychol. Bull. 76:105-110.

Wolfenbarger, S. N., Massie, S. T., Ocamb, C., Eck, E. B., Grove, G. G., Nelson, M. E., Probst, C., Twomey, M. C., and Gent, D. H. 2016. Distribution and characterization of Podosphaera macularis virulent on hop cultivars possessing R6-based resistance to powdery mildew. Plant Dis. 100: 1212-1221. 